Article

\title{
Exploiting the In-Network Capabilities of Multicast to Discover Proximate IPTV Channels
}

\author{
Chamil Kulatunga *, Jesse Kielthy, Dmitri Botvich and William Donnelly \\ Telecommunications Software and Systems Group, Waterford Institute of Technology, Cork Road, \\ Waterford, Ireland; E-Mails: jkielthy@tssg.org (J.K.); dbotvich@tssg.org (D.B.); \\ wdonnelly@tssg.org (W.D.)
}

* Author to whom correspondence should be addressed; E-Mail: ckulatunga@tssg.org; Tel.: +353-51-302971.

Received: 11 August 2010; in revised form: 24 September 2010 / Accepted: 25 September 2010 / Published: 29 September 2010

\begin{abstract}
IPTV has become the next generation of television due, in part, to its ability to support features that have been lacking in conventional broadcasting-for example, end-user interactivity, personalisation and localisation. Providers are also searching for the most efficient delivery methods to provide the greatest amount of contents at the lowest cost. At present IPTV uses IP multicast to deliver live TV channels in an over-provisioned walled-garden network due to issues of deploying multicast and QoS challenges in the public Internet. However, IPTV is likely to shift into some parts of the public Internet in the future as a managed service. Multicast routing is performed on a per-session destination-address basis so each router maintains a table of all of the multicast addresses to which the content is being forwarded. We exploit this information to discover and join the in-progress channels of geographically proximate users and to create a new incentivised premium service in future IPTV networks called ProxyTV. This approach is expected to minimise network bandwidth requirements as it enables ISPs to optimise bandwidth on their edge networks. This becomes increasingly significant as TV content consumes more and more bandwidth, especially with the onset of HD and 3D capabilities. In this paper, we have presented in detail the concept with the results of a survey and an analysis of network traffic to justify the proposed approach.
\end{abstract}

Keywords: IP multicast; IPTV; channel proximity; user-provided networking; social TV; traffic localisation 


\section{Introduction}

Even though the Internet has brought many new entertainment opportunities, watching television remains as one of the primary leisure activities around the world (average TV watching time per person is 24 hours per week in North America [1]). Internet Protocol TV (IPTV) has given new dimensions to the television experience with advanced features such as interactivity, personalization and localization [2] that conventional TV broadcasting is unable to support. Also, the deployments of equipment (for network operators), services (for service providers) and contents (for content producers) have become increasingly simplified in an IP environment. ITU-T [3] and ETSI-TISPAN [4] are working towards architectural frameworks and IETF is continuously engineering the TCP/IP protocols suitable also for video communications. IPTV is currently deployed in walled-garden networks using multicast to deliver live (linear) TV channels whereas Video on Demand (VoD) uses unicast, which delivers pre-recorded programs to the IPTV users in the same networks.

Numbers of IPTV subscribers and bandwidth requirements of the contents with High-Definition (HD) and 3-Dimensional (3D) video are increasing day by day at a higher rate than the technologies for network bandwidths are improved. As a result over-provisioned walled-garden IPTV networks may become congested in the recent future. In another way some parts of the public Internet will be multicast capable to deliver IPTV subjected to the challenge of Quality of Service (QoS) guarantee. QoS provisioning in the future Internet is not about commissioning hardware resources endlessly. Therefore we need to address this issue by optimally managing the network resources.

In IP multicast (also called native multicast), a single packet from the source is delivered to multiple receivers where multicast enabled routers duplicate packets as needed (creating a tree). This reduces traffic in the network when simultaneously communicating to a group of users. IP multicast standards have evolved by the IETF since 1989 when it was first introduced by S. Deering [5]. Due to deployment issues like scalability, congestion control, reliability and security, it has not been made available end-to-end over the public Internet but has been deployed in research networks like Internet2 MBone [6] and by some ISPs like JANET. After a long time in research, development and standardisation, IPTV today has grasped the advantages of multicast.

A multicast session is uniquely identified by a multicast address and routing is based on this multicast address in the destination address field (i.e., in contrast to the destination network-prefix based routing in unicast). As a result, each multicast router should maintain the multicast addresses of all the sessions that are flowing through it. We propose to exploit the routers' session information in a controlled way so that an end-user can discover other multicast sessions in its network proximity and reduce network traffic by joining with them. This paper analyses in detail the requirements for using this in-network capability of multicast to discover proximate sessions and develops a new socio-economic service paradigm for the benefit of end-users and IPTV providers. This novel system called ProxyTV generates incentives to encourage users to join an existing multicast session (i.e., a linear TV channel), which is closer (in network proximity) to a user, rather than watching a new channel. This can drastically reduce traffic in IPTV networks and, in turn, provide enhanced Quality of Experience (QoE) for the end-user.

Multicast service discovery using centralized servers where end-users explicitly inform about the sessions has been a research topic for a long time. However, it does not provide any information about 
geographical proximity. P. Harsh et al. [7] has proposed an approach to integrate geographical information into multicast service discovery without using more precise router information, which is related to our proposed concept. Their proposal leverages multicast Domain Name Service (DNS) architecture by tagging multicast sessions with geographical and spatial information based on the channel content or the service provider location. Internet Group Management Protocol (IGMP) spoofing [8] tracks the active multicast sessions only in a LAN domain where we propose to extend that into the routing domain.

The rest of the paper is organized as follows. In Section 2, we describe the requirements of collectively watching real-time contents to handle emerging traffic in the Future Internet (FI). The proximity discovery system using multicast is explained in detail in Section 3 while Section 4 presents the results of a survey and an analysis of network traffic in support of the proposed system. Section 5 discusses the opportunities created by the ProxyTV concept in some areas for the FI and Section 6 concludes the paper.

\section{Requirements of Collectively Watching Real-time Contents}

With wideband optical modulation techniques like D-WDM, the core of the Internet (mainly fibre-optics with OC-192/768 SONET) is observed to be congestion free (currently a considerable percentage of the Internet core fibres are believed to be dark) and it is trouble-free and economical for expansions. Also, access network technologies like VDSL2, FTTH, WiMaX and IEEE 802.11n ensure affordable fast last-miles. However, edge networks (i.e., networks connecting access networks to the core, Figure 1) could remain a bottleneck, especially with the onset of bandwidth intensive applications like HD and 3D video. Such links are usually over a long distance and network providers face many difficulties in installing and maintaining the underground fibre-optic cables like OC-1/3. In many cases, long-distance radio links such as T1/E1 may be used. It has also been experimentally determined that congestion bottlenecks mostly exist in the Internet edge networks [9].

Figure 1. Existence of an edge network bottleneck.

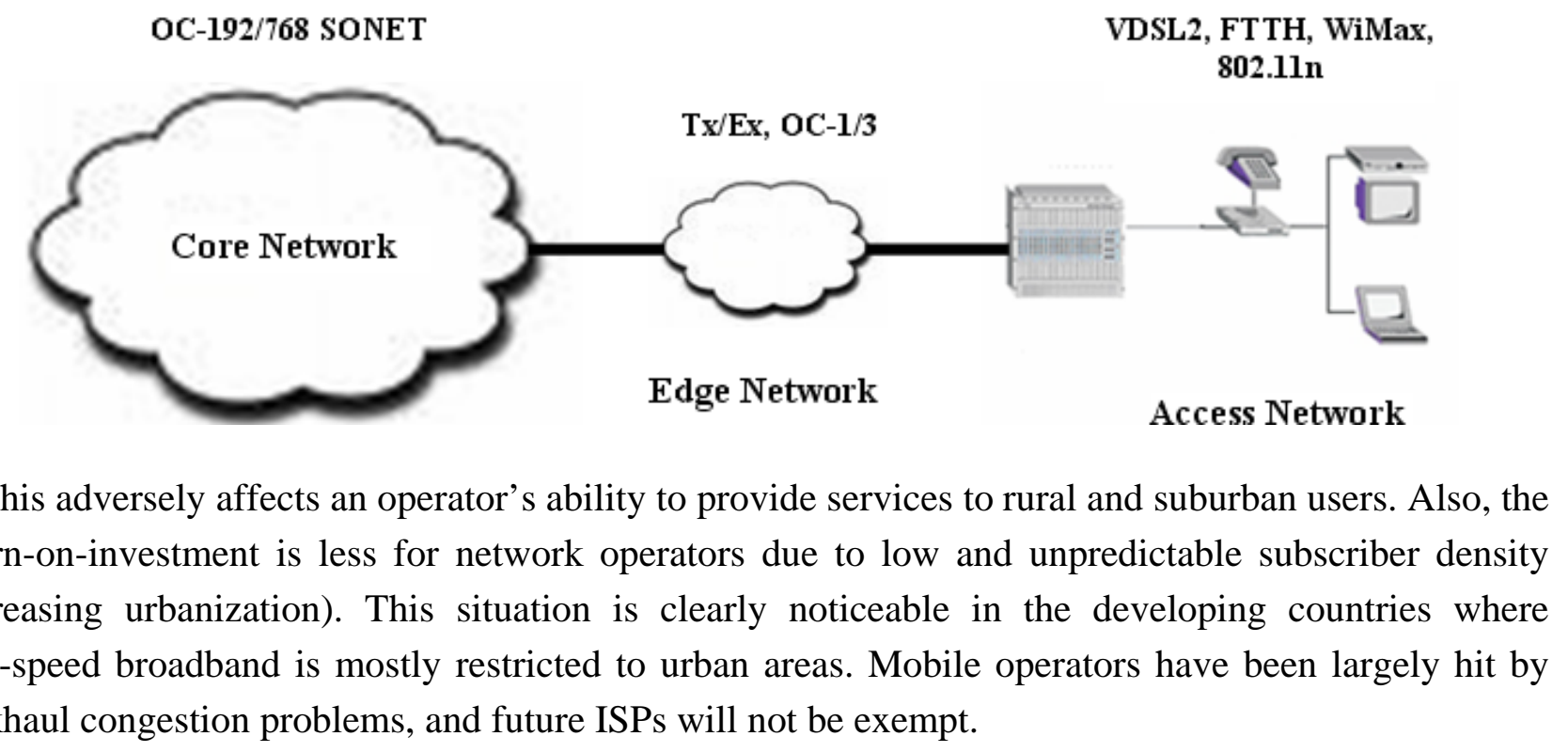


In VoD using unicast, a congestion control mechanism can be used without degrading the performance to the end-users (i.e., smoothly responsive DCCP or even aggressive TCP) by large data buffering. By contrast, multicast congestion control remains an open issue and therefore QoE degradation in live channels will become a grave concern for IPTV service providers where such channels anticipate a large number of concurrent participants. Current IPTV networks have, to a large extent, national coverage and mostly watched during the peak hours of 17:00 to 22:00. As a result, ISPs may look into prioritising all or a set of multicast channels using QoS mechanisms like Differentiated Services (DiffServ) [10].

In networks where such QoS is provided, premium service bandwidth is a scarce resource from the network operator's point-of-view and expensive service from user's point-of-view. This is because strict admission control is applied and the network may be over-provisioned. With the edge network bottlenecks, ISPs may not be able to simultaneously guarantee bandwidth throughout the network to satisfy all of its live channels or subscriber will not be able to afford for such premium channels (if over-provisioned). This may also result in IPTV providers limiting the number of available channels.

Therefore, network providers need to look at solutions to tackle this problem by managing bandwidth effectively at edge networks. ProxyTV approach is considered as a solution for the problem in future managed IPTV delivery over the public Internet in oppose to current over-provisioned walled-garden IPTV networks.

\section{Channel Proximity Discovery}

The first part of this section provides a brief explanation about route establishment and data forwarding in IP multicast. Then the novel system of channel proximity discovery using IP multicast is presented in detail.

\subsection{IP Multicast}

In creating a multicast forwarding tree rooted at the sender (dynamically changed according to the locations of the receivers in the network), receiving hosts join and leave a group from a LAN using the IGMP protocol [11]. Multicast routing protocol (Protocol Independent Multicast-Sparse Mode (PIM-SM) [12] is by far the most commonly used) grafts and prunes the branches based on the availability of the receivers in the IGMP domain.

Each multicast router maintains a table of multicast addresses (with the corresponding interfaces), called Tree Information Base (TIB)/Multicast Routing Information Base (MRIB), to appropriately replicate data packets in the forwarding plane. Forwarding is based on a matching of the destination multicast address in the IP header with an entry in the MRIB. The philosophy of the use of multicast is that the network traffic does not increase in the uplink as the number of participants' increases in the downward network.

As illustrated in Figure 2, user A has joined with the multicast group 232.0.1.1 (using IGMP) which delivers content of channel-1. Then PIM-SM establishes a forwarding path from source to the destination A keeping the forwarding states at the routers $\mathrm{X}$ and $\mathrm{Y}$. Multicast data packets are forwarded based on the destination address 232.0.1.1 in the IP header and a matching in the MRIB. When user $\mathrm{B}$ joins with this group, the PIM-SM join message reaches to router $\mathrm{X}$ and it adds the 
corresponding interface $\mathrm{I}_{\mathrm{X} 2}$ to the already existing entry for destination 232.0.1.1 in MRIB. When a packet reaches to the router $\mathrm{X}$, it replicates and sends two separate packets to A and B respectively through the interfaces $\mathrm{I}_{\mathrm{X} 1}$ and $\mathrm{I}_{\mathrm{X} 2}$. However, the second join by user $\mathrm{B}$ does not incur extra traffic to the network between the source and the router $X$. Similarly user $C$ has joined with the channel-2 (232.0.1.2) via the multicast routers $\mathrm{Z}$ and $\mathrm{Y}$.

Figure 2. Routing in IP multicast.

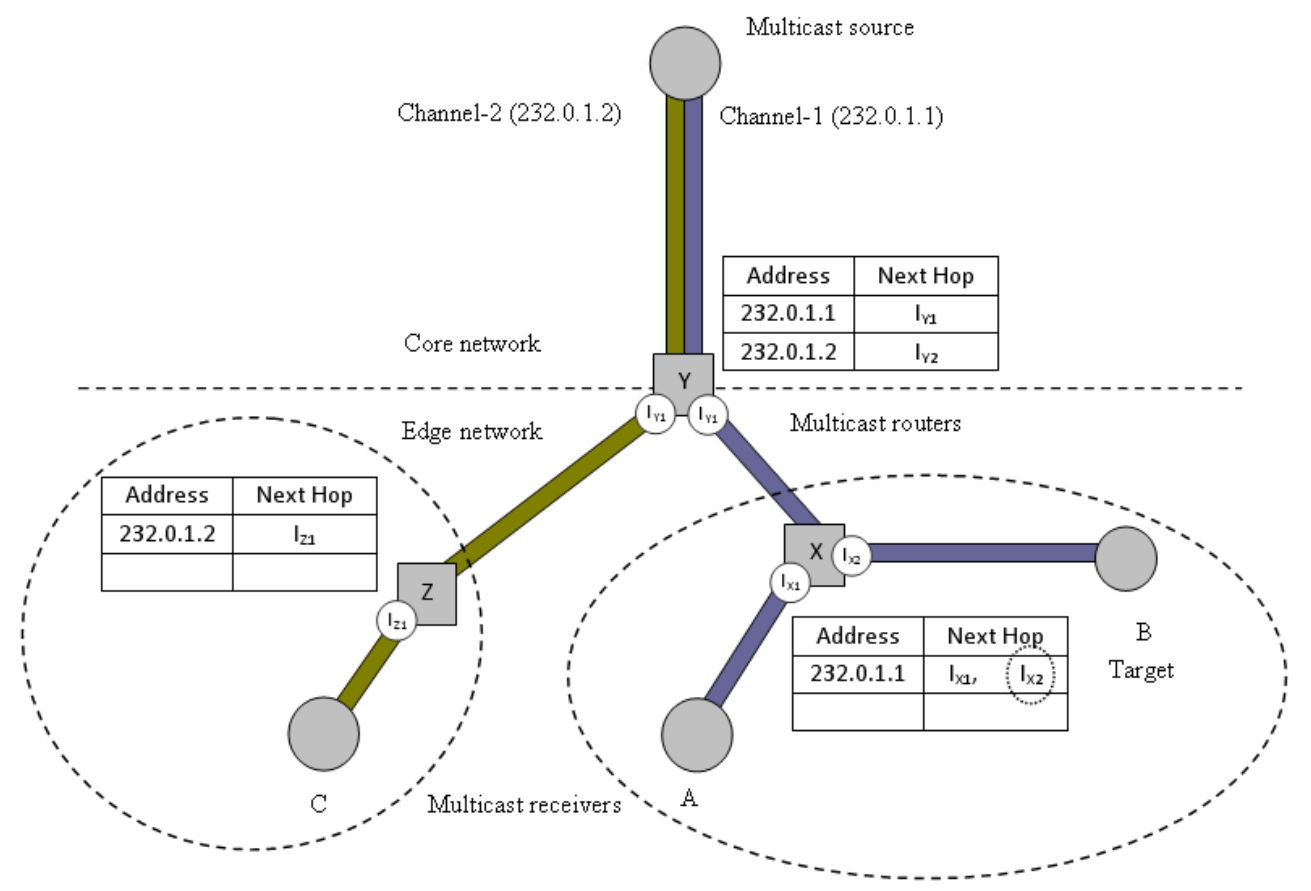

\subsection{The System of Channel Proximity Discovery}

As described in the above section, each router maintains the per-flow status of the multicast groups it forwards. The proposed system facilitates authorized end-users to exploit this information in multicast routers to discover the proximate in-progress channels. From this information, other users can subscribe to any of these channels without any additional traffic to the upstream network. We propose a user-initiated mechanism to use this in-network capability of multicast networks to allow users to discover the proximity of these channels without explicit notifications from other users.

The proposed user-initiated process of proximity discovery in IP multicast is shown in Figure 3 that can be broadly characterized as a pull-based approach. A user can periodically query nearby multicast routers (step 1) and join with any interesting proximate group. However, the routers maintain the multicast addresses in numerical format, where these addresses need to be resolved to user understandable names (i.e., using a network name resolution service similar to DNS for WWW) before a user can decide which channel they would like to subscribe. For this purpose, the user sends the queried addresses to a proposed Proximity Name Service (PNS) server as in step 2. Multicast application providers, who would opt to participate in this new service paradigm, are expected to register their TV channels with the PNS. Resolved addresses (i.e., named TV channels) can be announced to the user by means of Electronic Program Guide (EPG) or inserted advertisements. Users can subscribe to any proximate group that interests them (step 3). 
Figure 3. Query steps of the proximity discovery system.

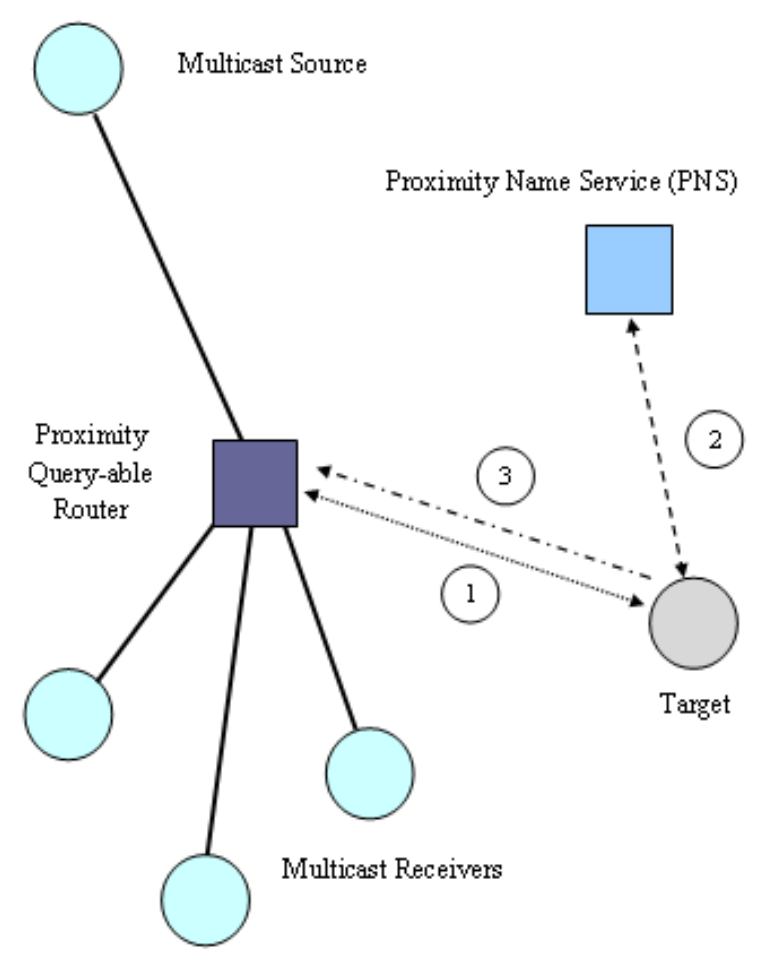

One PNS can be placed in a large geographical area as proximate channel announcements only target a requested user. Control traffic overhead can further be minimised allowing only qualified members to query and only registered channels to be responded at a proximity query-able router. The system can be implemented in a hierarchy as well. At the leaf router a user may find lesser number of in-progress sessions but these may be closest to the user. However, if a user can query other routers (higher up in the tree), they could potentially join more proximate sessions, though these would be found further into the network.

As shown in Figure 2, user A has joined with channel-1 first. Therefore the multicast address of channel-1 should be in the forwarding table of router X. Then the user B's STB (through periodic queries) will read this address and send to the PNS to resolve it. The resolved information will be available to the user B. Then user B can join with channel-1 without any extra traffic introduced to the network before router X. If user B's STB is allowed to query router Y, it will see both channel-1 and channel-2 are close by. However, changing to channel-2 introduces more traffic to the network (distant than channel-1).

An end-user querying the multicast table of a router which forwards data to many users, brings scalability, privacy and security issues. We propose to re-allocate a set of Source Specific Multicast (SSM) addresses from the IPv4 range 232.0.1.0 to 232.255.255.255 (In IPv6, this range is from FF3X::8000:0 to FF3X::FFFF:FFFF) [13], which has been allocated by IANA to dynamically use, when supporting proximity discovery for managed multicast applications. Then, a multicast router will respond with the ongoing multicast sessions that belong only to the assigned range. If an application provider decides to preserve the privacy of the users, like adult TV channels, they can avoid using addresses from the reserved range. This also reduces the size of the list of multicast addresses a router should respond to a user. 


\subsection{QoS Business Model}

In the proposed proximity discovery method, end-users should be influenced to select a TV channel that another proximate user is watching than joining with a new channel that the proximate router currently does not forward. Therefore the approach needs to be incentivised for the benefit of the end-user.

Assume the ISP guarantees QoS (DiffServ EF) for a set of HDTV channels and the SDTV channels are served under the best-effort service. Those HDTV channels are charged for a user at a certain cost per program and all the SDTV channels go under his/her monthly subscription. Then the first user can select a HDTV channel assuming others will join subsequently to share the cost (the first user confidence will grow up with the popularity of the system). The second user can see the proximity of the first user and may join knowing that they can definitely avail of a discount. The system needs to record the number of users per channel at a proximate router and apply a pricing model which reduces the cost of collectively accessing the content.

The FI is evolving as an in-network capable service-oriented architecture and network operators are expected to implement various services in addition to transporting the data as of today (i.e., aggregation routers will be integrated with service functionalities as well). Therefore in IPTV networks deploying ProxyTV, providers can allow subscribed users to query multicast routing information of selected edge routers (e.g., may be at the DSLAM). Video head-end can deploy PNS and feed the information of resolved TV channels to local delivery points.

\section{Performance Evaluations}

We have conducted a survey to identify a business model validating the proposed ProxyTV concept. Multicast traffic in a DiffServ network has been statistically analysed using the parameters derived from the survey.

\subsection{Survey Results}

The objective of the survey was to find out at what level of cost per program a user decides to wait for a proximate user to join considering the financial benefit to share the cost. After this value and if no other user has joined, the user may decide not to watch the intended program at the specified cost. We have conducted the survey with 45 users (only to derive some representative statistics) in two ranges of the costs based on the nature of the QoS guaranteed HD content; non-premium and premium. We have selected participants from Ireland and UK. They all pay for TV channels and have a good understanding of the concept of paying for TV based on the usage for specific contents on top of the monthly subscription. As an example they had additionally paid for some premium channels for a certain period at considerably higher rates like major sport tournaments. We explained to them about the benefits of collectively watching real-time contents with neighbours to gain economical incentives under a usage-based charging scheme. We suggested to them to think of one of their favourite programs under each category and complete the survey accordingly.

Figure 4a shows the results for a non-premium content like previously released movies, news, soaps, kids programs etc. A user's desire to wait for proximate user to join was enquired 
from 0.1 to 5 Euros. This is a reasonable range of rates for this kind of contents when derived from monthly subscription and the average monthly watching time of a person (but watching using HD) we have surveyed. According to the graph more than $80 \%$ of users say they will wait for a proximate user to join when the cost is approximately above 2.1 Euros. This shows their desire to use ProxyTV when the cost factor becomes significant.

Figure 4. Survey results (45 users). a. (top) A non-premium content; b. (bottom) A premium content.
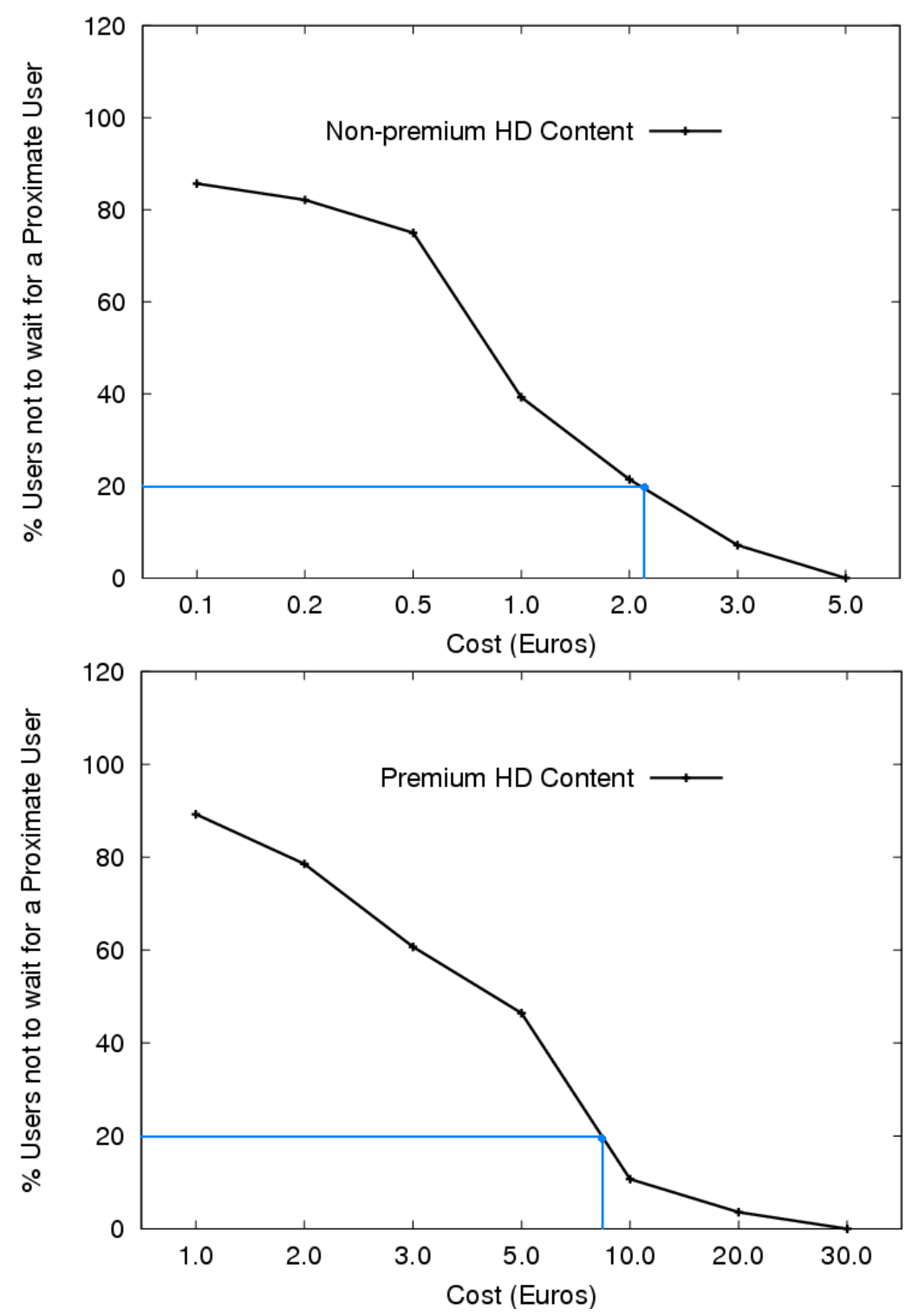

Figure $4 \mathrm{~b}$ shows the same results for a premium content like newly released movies, premium sport events, adult contents etc. The enquired rates for this category of contents were from 1 to 30 Euros. We have selected a higher range for premium contents considering the high cost (for high cost channels, cinemas, DVDs, etc.) they pay for watching premium HD contents intended to deliver in ProxyTV. In this case, more than $80 \%$ users opt to wait for a proximate user to join when the cost is approximately above 8.7 Euros. 


\subsection{Traffic Analysis}

We have conducted a statistical traffic analysis based on the survey results. We used 20 iperf UDP multicast sessions at 15 Mbps (i.e., a reasonable bandwidth for HDTV) over Linux implemented DiffServ EF (Expedited Forwarding) service [14]. Experiments have been conducted for two bottleneck link bandwidths; 100 Mbps and 200 Mbps (i.e., the bandwidth of the EF service over a link in the edge network shown in Figure 1). Each multicast session was active for $600 \mathrm{~s}$ at the server. However, if no user has joined with a channel there was no traffic from that multicast session over the bottleneck link.

Usually popularity distribution of video content is considered as a long-tail distribution and more precisely assumes a zipf distribution $[15,16]$. This indicates that popularity is high for few top channels and decays fast for non-popular ones. For simplicity, such a distribution abides by the 20-80 rule-that means $20 \%$ of the contents are watched by $80 \%$ of the users. Therefore we have selected 4 channels as very popular (80\% users watching) and remaining 16 as less popular (20\% watching). Within each category a user selected a channel with equal probability (i.e., creating a discrete step-wise distribution).

UDP and TCP short-lived unicast flows applied as background traffic (simulating VoD) also using the EF service. UDP (10 flows at 2 Mbps) and TCP (10 flows with window size of 85 KBytes) unicast flows were applied with random durations $(0 \ldots 600 \mathrm{~s})$ at random start times $(0 \ldots 600 \mathrm{~s})$. Packet size of all the flows was 1470 Bytes and a RED queue of size 400 Kbytes (RED parameters: min-thresh 100 Kbytes, max-thresh 300 Kbytes, dropping probability 0.1) was used for the EF class. Under the ProxyTV traffic analysis, we have chosen (according to the survey) that more than $80 \%$ of the users wait for another user to join (i.e., if IPTV provider decides the rates to 2.1 Euros for non-premium content and 8.7 Euros for premium content). Each experiment was repeated for 10 seed-values.

We have only considered joining behaviour of the users to demonstrate persuasiveness. The leaving behaviour has not been considered and therefore a user who joined at the beginning was active in the session throughput $600 \mathrm{~s}$ duration. In the both TV scenarios (marked as StdTV and ProxyTV in the graphs), all users joined a channel during a limited time at the beginning.

Figure $5 \mathrm{a}$ and $\mathrm{b}$ shows respectively the average values of receiving rate and packet loss rate of the multicast sessions at each user through a link bandwidth of 100 Mbps with and without the ProxyTV concept in place for different number of users. Figure 5c shows the number of multicast sessions (corresponding to IPTV channels) consumed the DiffServ EF bottleneck in this scenario with 90\% confidence intervals.

Under StdTV with 5 users, an average of approximately 4.0 sessions is used and it receives at around 13 Mbps rate. When the numbers of users are increased, the receiving rate degraded due to sharing of the bottleneck by many multicast sessions (reaching almost all 20 channels with more than 300 users). With ProxyTV, the number of multicast sessions at a time is significantly less than that of StdTV from 5 to 800 users. The decline of the receiving rates is not as drastic as with StdTV. ProxyTV approach only reaches the saturation point of 20 channels with more than 800 users.

According to the graphs, an ISP can statistically achieve considerably lesser packet loss rate in the service as a QoE parameter even at one third of the maximum link bandwidth required for all the channels (i.e., $20 \times 15 \mathrm{Mbps}$ ) with ProxyTV in compared to StdTV. 
Figure 5. Statistical performances with and without ProxyTV (100 Mbps link bandwidth). a. (top) Receiving rate; b. (middle) Packet loss rate; c. (bottom) Multicast sessions.
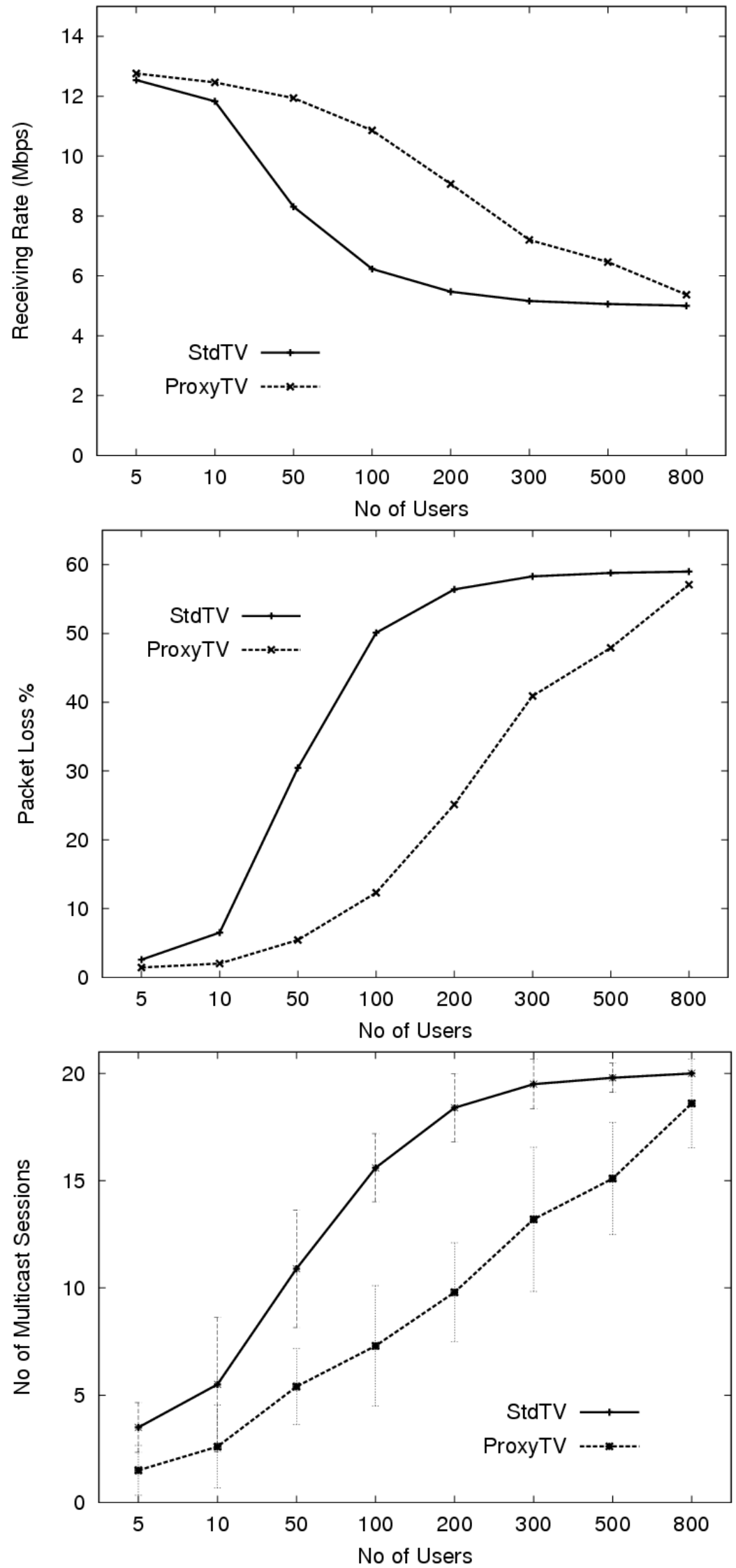
Figure 6. Statistical performances with and without ProxyTV (200 Mbps link bandwidth). a. (top) Receiving rate; b. (middle) Packet loss rate; c. (bottom) Multicast sessions.
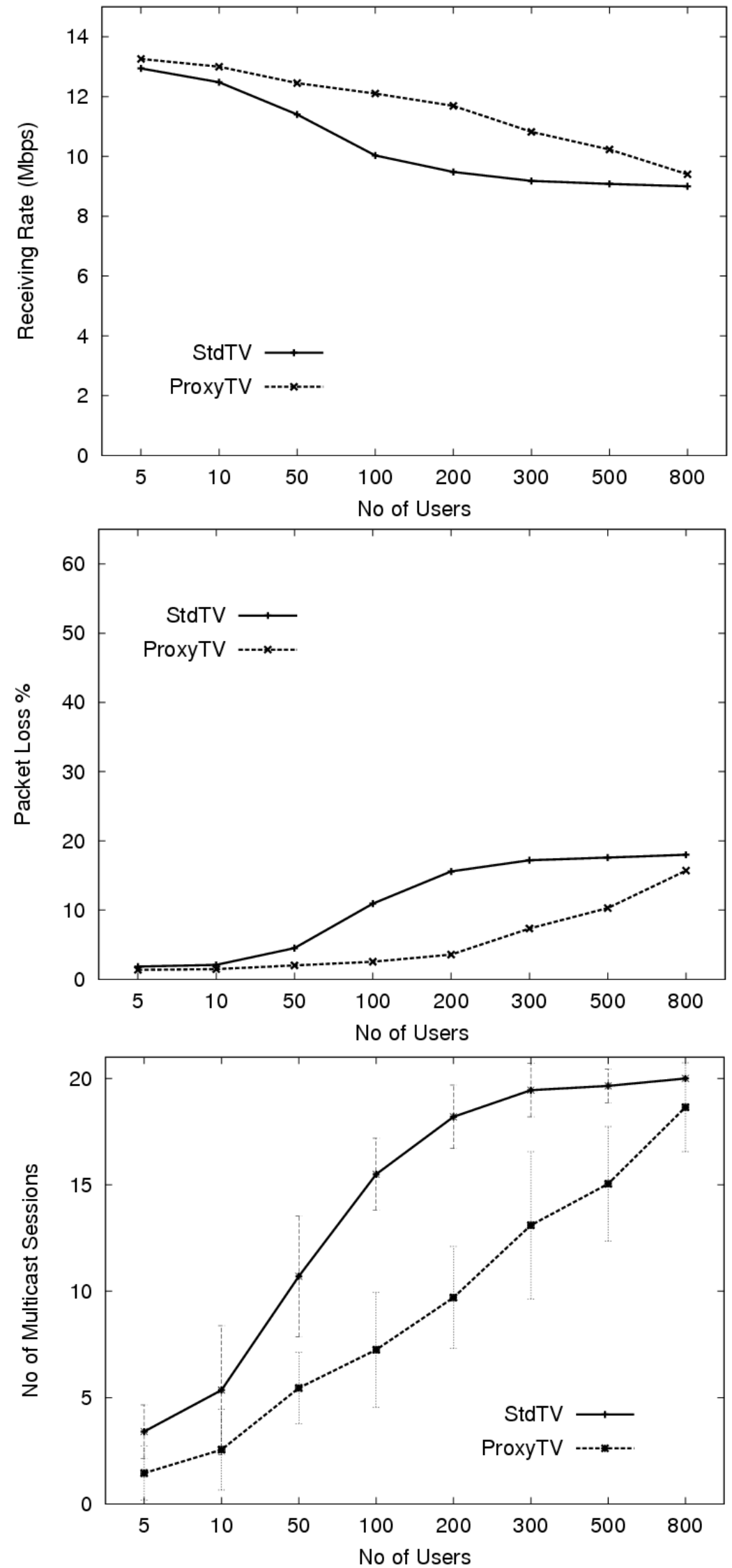
Figure 6 shows the same results for a link bandwidth of 200 Mbps with and without the ProxyTV approach. According to Figure $6 \mathrm{~b}$, an ISP can archive an acceptable packet loss rate $(<5 \%)$ in the service up to 250 users even at two third of the required maximum link bandwidth for all the channels with ProxyTV. To achieve the same loss rate, it can only serve up to 50 customers using StdTV. Figure 6c shows the number of multicast sessions that consume the DiffServ EF bottleneck for this link bandwidth is also significantly lower for ProxyTV than for StdTV.

\section{Opportunities for the Future Internet}

We have identified three future Internet perceptions that support the approach of the proposed innetwork service proximity discovery. These perceptions are expected to help implement ProxyTV beyond the current IPTV networks and allow the concept to grow and create many more novel network services.

\subsection{User-provided Networking}

A conventional residential networking paradigm was based on the concept that a router usually belongs to the ISP. This concept was changed with Home Area Networking (HAN) where the routers belong to the end-user. User-Provided Networking (UPN) [17] changes this model further from "my router" to a model of "our router" where a router might belong to a group of users in a community where resources can be shared among the users to build island-networks. In UPN, a group of users share network resources to communicate each other and to connect to the Internet. UPN provides new opportunities for proposed proximity discovery service enabling a community to use common network resources where traffic optimization becomes partially a users' responsibility (i.e., QoE needs to be earned).

There are some comparative initiatives such as FON [18], a commercial WiFi provider, which has sold millions of wireless access-points to their customers worldwide so that the community can share Internet connectivity among themselves. Similarly IPTV providers can introduce community shared routers at the user-end to implement the proximity discovery service. This also resembles the situation whereby mobile operators sell mobile phones to a group to allow them to form their own community reducing the costs among the users. Commercialized femtocell base-stations [19] (using an IP backhaul) for mobile users will also support the philosophy of this new service.

\subsection{Social TV Perspectives}

Delivery of TV channels can be innovative based on the common interests of a niche communitysuch delivery mechanisms are called Social Television (SoTV). These proposals have developed technologies to increase interactivity among users. Geographical locality has not been a factor in interactivity but naturally many common interests can be based on geography in order to allow face-to-face interactions. As an example, students might discuss about an educational program in the classroom they watched at home. Also the traffic locality requirement becomes vital with the high-bandwidth applications in the public Internet. It can improve user's QoE using multi-party 
communication approaches. Therefore, SoTV can also be used for network traffic localization objective moving beyond the interactivity feature.

Ambient TV [20] suggests using a second screen to visualize people and programs they are watching. It improves the speed of user reactions without disturbing the ongoing program. Such mechanisms could become important for implementing ProxyTV where, using pervasive sensing, we can visualise the proximate channels when watching a certain program. Users may join with popular programs frequently watched by many proximate users within the community. IPTV has already developed technologies to feed localized advertisements to a targeted group of people [21]. This could be used as a mechanism to attract new users into the proximate user society.

\subsection{Trends in $P 2 P$}

Network providers are looking for local traffic optimization techniques for the benefit of both Internet users and providers. Particularly, with the onset of $\mathrm{HD}$ and $3 \mathrm{D}$ in the near future, high-bandwidth services should become prime candidates for traffic localization. The IETF Application Layer Traffic Optimization (ALTO) working group is standardising this aspect for the P2P approach [22]. ALTO proposes a network-assisted P2P framework where P2P applications use information provided by ISPs in best peer selections. It resembles to that circuit-switched telecom operators maximise traffic within their own networks through economic incentives to the users.

Therefore, the ProxyTV approach and in general the session proximity discovery capability of multicast can be used by the network providers to localise traffic subjected to widespread deployment of IP multicast.

\section{Conclusions}

Even though the IPTV has restricted to over-provisioned walled-garden networks today, it is likely to deliver this multicast enabled service completely or partially using the public Internet in the near future. It is also unavoidable to investigate new approaches to optimally use existing network resources to handle increasing bandwidth demand of rich media contents due to the limitations of throwing out additional network capacities. We have proposed a new network service (by exploiting the session information at the multicast routers) to discover and join with live multicast TV channels those are being watched by the proximate users. A business model has also been proposed to provide incentives for users to watch TV channels communally. The concept has been strengthened by our survey results which show that TV users are eager to share the cost when the price of the content exceeds a threshold. Our traffic analysis proves that it is a win-to-win situation for IPTV providers as well by significantly reducing the network traffic. The paper has also discussed the motivations offered by the emerging FI tendencies like UPN, SoTV and ALTO towards the proposed proximity discovery approach.

\section{Acknowledgements}

This work was supported by the project "FutureComm: Serving Society" funded by the Higher Education Authority (HEA) in Ireland under the Programme for Research in Third-Level Institutions (PRTLI) Cycle 4 and this work was also undertaken in the context of the Magneto-Celtic 
Telecommunication Solutions Project, which is partially funded by the Commission of the European Union.

\section{References}

1. American Time Use Survey, 2008. Available online: http://www.bls.gov/tus (accessed on 25 September 2010).

2. Xiao, Y.; Du, X.-J.; Zhang, J.-Y.; Hu, F.; Guizani, S. Internet Protocol Television (IPTV): The Killer Application for the Next-Generation Internet. IEEE Commun. Mag. 2007, 45, 126-134.

3. ITU-T IPTV Focus Group. Available online: http://www.itu.int/ITU-T/IPTV (accessed on 25 September 2010).

4. ETSI Telecoms \& Internet Converged Services \& Protocols for Advanced Network (TISPAN). Available online: http://www.etsi.org/tispan (accessed on 25 September 2010).

5. Deering, S. Host extensions for IP multicasting. IETF 1989, RFC 1112.

6. Diot, C.; Neil, B.; Bryan, L.; Balensiefen, K.D. Deployment Issues for the IP Multicast Service and Architecture. IEEE Netw. Mag. 2000, 14, 78-88.

7. Harsh, P.; Newman, R. Using Geo-spatial Session Tagging for Smart Multicast Session Discovery. In Proceedings of the ACM Symposium on Applied Computing (SIGAPP), Honolulu, HI, USA, March 2009.

8. Christensen, M.; Kimball, K.; Solensky, F. Considerations for Internet Group Management Protocol (IGMP) and Multicast Listener Discovery (MLD) Snooping Switches. IETF 2006, RFC 4541.

9. Akella, A.; Seshan S.; Shaikh, A. An Empirical Evaluation of Wide-Area Internet Bottlenecks. In Proceedings of the ACM International Conference on Measurement and Modeling of Computer Systems (SIGMETRICS), San Diego, CA, USA, June 2003.

10. Blake, S.; Black, D.; Carlson, M.; Davies, E.; Wang Z.; Weiss, W. An Architecture for Differentiated Services. IETF 1998, RFC 2475.

11. Cain, B.; Deering, S.; Kouvelas, I.; Fenner B.; Thyagarajan, A. Internet Group Management Protocol, Version 3. IETF 2002, RFC 3376.

12. Fenner, B.; Handley, M.; Holbrook H.; Kouvelas, I. Protocol Independent Multicast-Sparse Mode (PIM-SM). IETF 2006, RFC 4601.

13. IANA Multicast Address Assignments. Available online: http:/www.iana.org/assignments/ multicast-addresses (accessed on 25 September 2010).

14. Kulatunga, C.; Kielthy, J.; Malone P.; Foghlu, M.O. Implementation of a Simple Bandwidth Broker for DiffServ Networks. Presented at International Workshop on Inter-domain Performance and Simulation (IPS), Budapest, Hungary, March 2004.

15. Cha, M.; Rodriguez, P.; Crowcroft, J.; Moon S.; Amatriain, X. Watching Television over an IP Network. In Proceedings of the ACM Internet Measurement Conference (IMC), Vouliagmeni, Greece, October 2008.

16. Qiu, T.; Ge, Z.; Lee, S.; Wang, J.; Xu J.; Zhao, Q. Modeling User Activities in a Large IPTV System. In Proceedings of the ACM Internet Measurement Conference (IMC), Chicago, IL, USA, November 2009. 
17. Sofia, R.; Mendes, P. User-Provided Networks: Consumer as Provider. IEEE Commun. Mag. 2008, 46, 86-91.

18. FON WiFi Community. Available online: http://www.fon.com (accessed on 25 September 2010).

19. Ortiz, S. The Wireless Industry begins to Embrace Femtocells. IEEE Comput. Mag. 2008, 41, 14-17.

20. Harboe, G.; Metcalf, C.J.; Bentley, F.; Tullio, J.; Massey, N.; Romano, G. Ambient Social TV: Drawing People into a Shared Experience. In Proceedings of Conference on Human Factors in Computing Systems (CHI), Florence, Italy, April 2008.

21. Hart, T. The Benefits of Addressable Advertising in Broadcast (Linear) TV over Telco Networks. Packet Vision White Paper, 2008. Availabsle online: http://www.packetvision.com/dmdocuments/ Packet Vision_Business Case-Addressable Advertising for IPTV_Tony Hart.pdf. (accessed on 28 September 2010)

22. Gurbani, V.; Hilt, V.; Rimac, I.; Tomsu, M.; Marocco, E. A Survey of Research on the Application-Layer Traffic Optimization Problem and the need for Layer Cooperation. IEEE Commun. Mag. 2009, 47, 107-112.

(C) 2010 by the authors; licensee MDPI, Basel, Switzerland. This article is an open access article distributed under the terms and conditions of the Creative Commons Attribution license (http://creativecommons.org/licenses/by/3.0/). 